\title{
Percutaneous Alcohol Sclerotherapy of a Hepatic Hydatid Cyst after Balloon Occlusion of a Large Biliary Communication
}

\author{
Jozsef Varro $^{a} \quad$ Laji Mathew $^{\mathrm{a}}$ Reji Philip Athyal ${ }^{\mathrm{a}} \quad$ Abdulrahman H. Khafagy ${ }^{\mathrm{b}}$

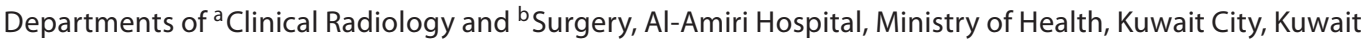

\section{Key Words}

Hepatic hydatid cyst · Percutaneous sclerotherapy •

Occlusion balloon catheter $\cdot$ Biliary communication

\begin{abstract}
Objective: To present a case of hepatic hydatid cyst with a biliary communication that was not suitable for surgery and hence necessitated sclerotherapy with absolute alcohol after occluding the biliary communication with a balloon catheter. Clinical Presentation and Intervention: A 50-year-old Asian man presented to the surgical emergency department with a 1-year history of repeated attacks of obstructive jaundice and right hypochondrial pain. Ultrasound and contrast computed tomography revealed a cyst, and endoscopic retrograde cholangiopancreatography and cystography revealed a biliocystic communication. An indirect hemagglutination test for echinococcosis showed the presence of antibodies to Echinococcus species at a titer of 8 establishing the diagnosis of hydatid cyst. Although surgery is the accepted modality of treatment in these cases, the patient was deemed unfit for surgery due to his underlying cardiac problem. While percutaneous treatment with absolute alcohol is contraindicated in his case, it was successfully attempted after balloon occlusion of the biliocystic communication. Con-
\end{abstract}

clusion: This case showed that in this patient with hepatic hydatid disease and biliocystic communication, who was not fit for surgery, percutaneous sclerotherapy with absolute alcohol after balloon occlusion was successfully performed by an interventional radiologist.

Copyright $\odot 2011$ S. Karger AG, Basel

\section{Introduction}

Minor communication of hydatid disease with the biliary tree has been described in $5-76 \%$ of hepatic hydatid cysts, which may be occult; however, frank biliary (large persistent) communication has been reported in only $5-15 \%$ of cases $[1,2]$. Surgery is the preferred mode of treatment in these cases, and percutaneous treatment with absolute alcohol is contraindicated due to the risk of chemical sclerosing cholangitis [3]. However, we present a case of hepatic hydatid cyst with a large biliary communication that was successfully treated by percutaneous sclerotherapy with absolute alcohol after balloon occlusion of the biliary communication. This was necessary as the patient was considered unfit for surgery and hence had no other treatment option. 
1

Fig. 1. Cystography shows irregular cyst wall with filling defects and biliocystic communication (open arrow) with opacification of the biliary tree with multiple filling defects (solid arrows) representing membranes of the hydatid cyst.

Fig. 2. Double contrast cystography shows pigtail catheter in the cyst with irregular walls (open arrow) and inflated balloon catheter placed across the biliocystic communication (solid arrows) prior to the alcohol ablation.

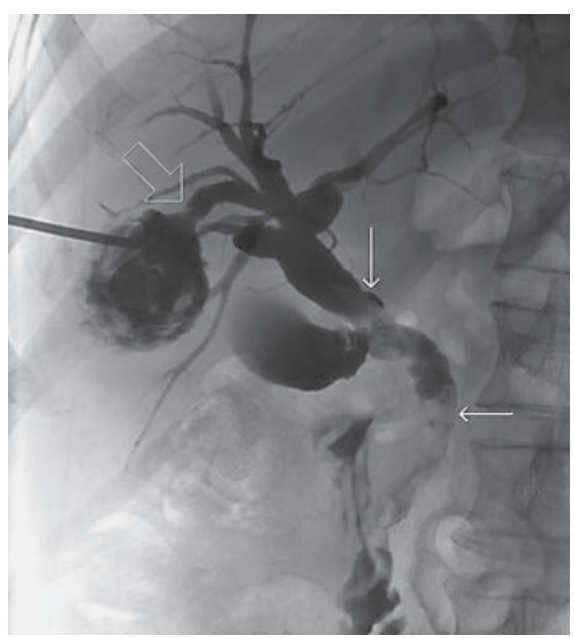

2

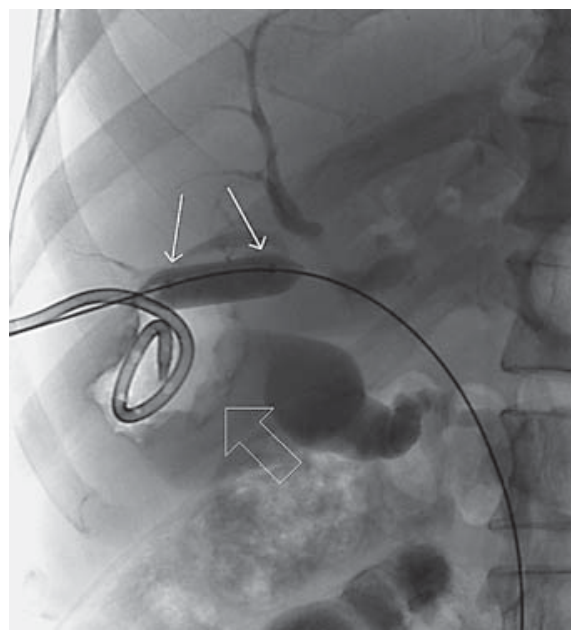

\section{Case Report}

A 50-year-old Asian man presented to the surgical emergency department with a 1-year history of repeated attacks of obstructive jaundice and right hypochondrial pain. Ultrasound revealed a well-defined rounded heterogeneous focal lesion in the right lobe of the liver measuring $8.5 \times 5.5 \mathrm{~cm}$ with an undulating hyperechoic inner wall and fine internal echoes. Contrast computed tomography revealed a cystic lesion with no enhancement. There was associated intrahepatic biliary dilatation. Magnetic resonance cholangiopancreatography showed mild intrahepatic biliary dilatation with no definite communication seen between the lesion and the biliary tree. An indirect hemagglutination test for echinococcosis showed the presence of antibodies to Echinococcus species at a titer of 8 . An endoscopic retrograde cholangiopancreatography showed faint contrast opacification of the lesion that was observed on ultrasound and computed tomography, suggesting a communication with the biliary tree. A papillotomy was performed during the procedure.

The patient had an attack of sinus ventricular tachycardia and a transthoracic echocardiography revealed grade 1 diastolic dysfunction, dilated left atrium, right atrium, right ventricle, and a large atrial septal-ostium secundum type defect with a diameter of $3.4 \mathrm{~cm}$. Although the biliocystic communication could be operatively managed, it was not done due to the patient's poor cardiac status and probable impending failure. The patient was then started on medical treatment with albendazole tablets $400 \mathrm{mg}$ twice daily.

The hydatid cyst was drained through a 10-French pigtail catheter under ultrasound and fluoroscopy guidance. At the beginning of the procedure, very little fluid could be aspirated from the cyst and when contrast medium was injected, small filling defects were seen in the cyst and biliary ducts (fig. 1). The communication between the cyst and one of the branches of the major right hepatic duct was revealed. With repeated catheter manipulation and after flushing with diluted contrast medium, more of the content was aspirated; the remainder was flushed into the biliary ducts and duodenum. This flushing was done to get rid of the solid particulate matter from the cavity in order to enhance contact with the sclerosing agent when the procedure was repeated thereby facilitating shrinkage of the cavity. At the end of the procedure, most of the solid parts of the cyst were flushed into the small intestine. The biliary ducts had no filling defects, and a large communication ( $6 \mathrm{~mm}$ in diameter) was clearly seen between the cyst and the right biliary duct.

Since a high volume of bile continued to drain through the percutaneous drainage, the patient needed a definitive treatment. Sclerotherapy of the communicating hepatic hydatid cyst was done through the existing access with an occlusion balloon catheter to prevent the passage of the sclerosing agent into the biliary system. The sclerotherapy was performed in two stages using absolute alcohol. Two guide wires were placed through the existing catheter into the liver cyst. One of them was used to insert the balloon catheter of $10 \mathrm{~mm} \times 3 \mathrm{~cm}$ into the connecting biliary duct to occlude it (fig. 2). The other wire was used to insert a new drainage catheter to perform the sclerotherapy. Contrast media (of identical volume as the sclerosing agent) and air were injected to confirm the blockage of the communication.

Samples of 10 and $15 \mathrm{ml}$ of absolute alcohol were injected into the cyst in two stages. The sclerosing/coagulation effect was immediately noticed after injecting the absolute alcohol. The absolute alcohol was reaspirated after $20 \mathrm{~min}$. At the end of the procedure, the cavity was flushed with normal saline to remove any trace of alcohol left behind and the balloon catheter was deflated and removed.

There was a relatively rapid initial reduction in bile drainage from 300-500 $\mathrm{ml}$ per day with eventual complete cessation of drainage over a period of 24 days. A follow-up computed tomography showed complete resolution of the hydatid cyst and absence of previously noted intrahepatic biliary dilatation. The drain was closed for $48 \mathrm{~h}$ and repeated ultrasound showed no fluid accumulation at the site of the sclerosed hydatid cyst. The drainage catheter was then removed and the patient discharged. Unfortunately, further follow-up was not done because the patient, who was an expatriate, had left for his home country and has not returned as to date. 


\section{Discussion}

In complicated hydatid disease of the liver, biliary communication occurs with the right duct in $55-60 \%$ of cases, the left duct in 25-30\%, and rarely at the confluence or with the gallbladder [4]. In our case, the communication was with a branch of the right main duct. Communicating rupture can be a simple communication between small biliary radicles and the cyst, or frank rupture into the biliary tree [4] as in our patient. Endoscopic drainage can be performed by sphincterotomy, nasobiliary drainage, or stent insertion, alone or in combination $[5,6]$. Surgery is indicated in cysts of any type that show any form of complication, such as compression of the adjacent organs, jaundice and intrabiliary rupture [7]. However, an isolated report of 3 cases with biliary communication treated with a combination of cetrimide and hypertonic saline as sclerosant has been reported [3] similar to our case. In our patient, because of the presence of the large biliary communication with the sclerotherapy being performed after balloon occlusion, we felt that we need maximum effect of the sclerosing agent in a short time during which the balloon is occluding and hence we proceeded directly with absolute alcohol rather than attempting with a less aggressive agent like hypertonic saline which may not be effective. Percutaneous sclerotherapy with absolute alcohol is contraindicated in such cases without blocking the communication due to the risk of the sclerosing agent entering the biliary tree with resultant chemical sclerosing cholangitis [3].
Our case was high risk for surgery due to the underlying cardiac problems. Surgery also brings significant risk in the management of cystobiliary communications. Hepatectomy and pericystectomy are radical operations for hydatid liver cyst. Radical surgery carries a perioperative risk, but postoperative biliary leakage and recurrence are rare. Conservative surgery, which is preferred in endemic areas, carries a high incidence of postoperative biliary leakage and local recurrence [8]. In the absence of other treatment options, sclerotherapy with absolute alcohol was attempted after blockage of the biliary communication with an occlusion balloon. This was resorted to as we felt that coiling the communication prior to the sclerotherapy might not completely occlude the opening and surgical ligation was also not an option as mentioned above. Following the balloon occlusion, the sclerotherapy was performed using absolute alcohol with successful ablation of the hydatid cyst and thereby resolution of the biliary dilatation.

\section{Conclusion}

Though surgery still remains the primary modality for treatment of communicating hepatic hydatid cysts, it also brings significant risk in management and hence percutaneous sclerotherapy with absolute alcohol is a feasible alternative after occlusion of the communication.

\section{References}

$>1$ Mehmet I, Surreya S, Erol A, Ezici H: Percutaneous transhepatic endobiliary drainage of hepatic hydatid cyst with rupture into the biliary system: an unusual route for drainage. Cardiovasc Intervent Radiol 2002;25: 437-439.

-2 Ibrahim A, Serpil Y, Yazuz C, Resat K, Metin B: Fistulous communication between a hepatic hydatid cyst and the gallbladder: diagnosis with MR cholangiopancreatography. AJR Am J Roentgenol 2005;185:12111213.
$>3$ Haddad MC, Huwaijah SH, Al-Kutoubi AO: Re: the safe use of cetrimide and hypertonic saline for percutaneous ablation of hepatic echinococcal cysts complicated by intrabiliary rupture. Cardiovasc Intervent Radiol 2000;23:412-414.

4 Kumar R, Reddy SN, Thulkar S: Intrabiliary rupture of hydatid cyst: diagnosis with MRI and hepatobiliary isotope study. Br J Radiol 2002;75:271-274.

5 Ozaslan E, Bayraktar Y: Endoscopic therapy in the management of hepatobiliary hydatid disease. J Clin Gastroenterol 2002;35:160174.
6 Sharma BC, Agarwal N, Garg S, Kumar R, Sarin SK: Endoscopic management of liver abscesses and cysts that communicate with intrahepatic bile ducts. Endoscopy 2006;38: 249-253.

7 Menezes da Silva A: Hydatid cyst of liver criteria for the selection of appropriate treatment. Acta Trop 2003;85:237-242.

-8 Demircan O, Baymus M, Seydaoglu G, Akinoglu A, Sakman G: Occult cystobiliary communication presenting as postoperative biliary leakage after hydatid liver surgery: are there significant preoperative clinical predictors? Can J Surg 2006;49:177-184. 